Helmut Asche*

\title{
Europäische Handels- und Agrarpolitik gegenüber Afrika Mit einem kritischen Blick auf den Beitrag der Wirtschaftswissenschaften
}

https://doi.org/10.1515/zfwp-2018-0020

\begin{abstract}
European trade policy with Africa is in deep trouble. We observe a triple policy failure. (1) The EU tries to draw African partner countries into comprehensive deep integration agreements, far more than these countries can arguably support. (2) For trade in goods, safeguard clauses in the EPAs are patchy. They cannot satisfy African needs for smart protection of agricultural and industrial businesses. Facing the refusal of some African governments to sign, the EU has no answer. Ensuing fragmentation of African regional economic communities is a disaster. Rapid repair work of the existing regional EPA drafts looks more promising than a grand solution with the new African Continental Free Trade Area. (3) Negative impact of the EU Common Agricultural Policy on African producers must finally be acknowledged and at least partly compensated. - A deeply divided economic profession delivering trunked analyses with regard to all three complexes is unfortunately of limited help to policy-makers.
\end{abstract}

Globale Handelspolitik ist ein besonders erstaunliches Politikfeld. Im Rahmen der WTO wurden über Jahrzehnte extrem umfangreiche Politikpakete als sog. single undertakings verhandelt, bis darüber der Multilateralismus fast zum Stillstand gekommen ist und Regional Trade Agreements (RTA), die nach der WTODoktrin die Ausnahme sein sollten, wieder zum dominierenden Verhandlungsmodus geworden sind. In Handelstheorie und -politik wurden die Sozialfolgen globaler Handelsliberalisierung nahezu vollständig als vorübergehende Anpassungskosten abgebucht, bis wesentliche Akteure nun verstärkt aus dem System ausbrechen. Die gleichen Absonderlichkeiten setzen sich in rezenten bi-regionalen Verhandlungen fort - sowohl auf der Nord-Nord- als auch auf der Nord-SüdAchse.

\footnotetext{
*Kontakt: Helmut Asche, Johannes-Gutenberg-Universität und Institut für Ethnologie und Afrikastudien ifeas, Forum universitatis 6, 55099 Mainz, Germany, Tel.: (06131)39 22 798, E-Mail: asche@uni-mainz.de
} 
Aus der wissenschaftlichen Distanz betrachtet war es immer erstaunlich, mit welcher Unerschütterlichkeit deutsche Bundesregierungen, aber auch die deutsche Unternehmerschaft über ihre Spitzenverbände in den TTIP-Verhandlungen mit den USA ein Verhandlungskonzept mitgetragen haben, das so umfassend in Daseinsvorsorge und Selbstverständnis europäischer Gesellschaften eingriff, dass ein Scheitern vorprogrammiert war. Heute wissen wir, wie viel besser wir mit einem „TTIP light“ gefahren wären, das sich auf den Abbau der transatlantischen Zölle und technischen Handelshemmnisse konzentriert und so auch dem aktuellen Voluntarismus in der US-Handelspolitik entgegengewirkt hätte. Ähnlich verhält es sich nun mit den Handelsverträgen zwischen der Europäischen Union und den AKP-Staaten, insbesondere mit der subsaharischen Staatengruppe - mit den sog. Economic Partnership Agreements (EPAs). Wir beobachten ein dreifaches Politikversagen.

Erstens: Die deutsche Seite hat lange eine zweifelhafte Strategie der EUKommission unterstützt, die Afrika ebenso umfassende Abkommen wie TTIP aufnötigen wollte (und im Grunde immer noch will). Die afrikanischen Verhandler haben das nach zwölf Jahren EPA-Verhandlung korrigiert, unter dem Druck ihrer eigenen Zivilgesellschaft. Die nun ausgeklammerten Themenkomplexe sind auch als Singapur-Themen bekannt (Investitionsregeln, Wettbewerbspolitik, Öffentliche Ausschreibungen, Handelserleichterungen). Das Gleiche wiederfuhr den Themen Patentschutz und Dienstleistungen. Die provozierte Eingrenzung der EPAs auf die Regelung des reinen Güterhandels bleibt nun sogar hinter dem theoretisch Wünschbaren zurück: die vollständige Abtrennung von Verhandlungen über Güter und Dienstleistungen, einschließlich Logistik, IT, Finanzdienstleistungen, ist unter den Bedingungen globaler Arbeitsteilung nicht mehr realistisch. Wie die Bereitschaft afrikanischer Länder, in einer zweiten Runde auch über die Liberalisierung bestimmter Servicebereiche mit der EU oder den USA zu verhandeln, wiederhergestellt werden kann, bleibt realpolitisch schwer vorstellbar.

Das Misstrauen afrikanischer Verhandler gegenüber hoch aufgeladenen „WTO-Plus“-Verhandlungen auf bi-regionaler Ebene ist durch einen Teil der globalisierungskritischen Wirtschaftstheorie unterstützt worden. Hier begegnet uns zum ersten Mal ein spezifischer Dissens in den wirtschaftswissenschaftlichen Grundpositionen. Autoren wie Stiglitz et al. empfehlen, solche Nord-Süd-Verhandlungen „at all costs“ zu vermeiden, während der britische Mainstream der Collier, Venables et al. afrikanische Länder in Nord-Süd-Abkommen besser aufgehoben sieht als in ihren eigenen Süd-RTAs. Die Wirtschaftswissenschaften haben sich in dieser Sache kaum nützlich gemacht. Dass sie seit jeher über die Wohlstandswirkung wirtschaftlicher Regional-Unionen zerstritten sind, hat zunächst nichts mit Afrika oder anderen Entwicklungsregionen zu tun, sondern ergibt sich allgemein daraus, dass keine Einigkeit herrscht, ob Wirtschaftsgemeinschaften (und die Verträge 
zwischen ihnen) nun als stumbling blocks oder als stepping stones von globaler Wirtschaftsintegration zu betrachten sind. Die professionelle Skepsis gegenüber RTA in Afrika ist noch ausgeprägter - so sehr, dass Weltbank und Währungsfonds sie über Jahrzehnte unverhohlen abgelehnt und erst seit relativ kurzer Zeit beigedreht haben. Die Gründe für die Skepsis sind zum Teil fundiert, aber es hilft der politischen Sache nicht, dass es der Wirtschaftstheorie immer noch nicht gelungen ist, einen neuen Konsens zu finden, welche Themenfelder in welcher Fassung sich am ehesten für Regionalgemeinschaften in Afrika und für deren Nord-Süd-Vereinbarungen eignen. Das wirkt sich auch in den EPA-Verhandlungen aus.

Zweitens: Die EPAs sollen den Vertragsstaaten wie schon bisher zoll- und quotenfreien Zugang zum EU-Markt garantieren (nicht denen in Nordafrika, was ein eigenes Problem ist). Sie verlangen aber einen weitgehenden Zollabbau auch auf afrikanischer Seite. Dass solche im Kern reziproken Freihandelsabkommen in der WTO auch zwischen Industrie- und Entwicklungsländern Vorschrift und daher alternativlos seien, ist eine politische Mär. Verbleibende Zollsätze und Schutzregeln sind in den EPAs nun aber zu niedrig und zu unflexibel angesetzt, um den Aufbau wettbewerbsfähiger Industrien in Afrika zu fördern. Widerstand dagegen wird in Europa gern als „Protektionismus“ missverstanden, statt zu erkennen, dass heimische wie Auslandsinvestitionen in den meisten Wirtschaften Afrikas einen intelligenten und zeitlich begrenzten Außenschutz benötigen, um die schlechteren Standortbedingungen auszugleichen. Im transatlantischen Handel haben Zölle und Zolläquivalente keine Daseinsberechtigung mehr, wohl aber für Afrika. Hinzu tritt die Bedeutung von Zöllen als fiskalischer Hauptquelle in Afrika.

Auch zu dieser speziellen Fragestellung hat sich konventionelle wirtschaftswissenschaftliche Modellierung nicht verdient gemacht. Die EPA-Verhandlungen wurden über ihre gesamte Dauer von beauftragten Wirkungsstudien (ex ante impact assessments) begleitet. Sie beruhen auf partiellen oder allgemeinen Gleichgewichtsmodellen in Anwendung auf die afrikanischen Vertragsstaaten, neuerdings meist basiert auf GTAP 9. Die Studien vollständig zu dokumentieren, würde mehr als eine Druckseite füllen; nicht alle sind von der EU-Kommission bestellt. Ihr durchgängiges Ergebnis lautet:

(a) die Gesamtwirkung der Handelsliberalisierung im Zuge der EPAs wird relativ gering bleiben, meist schwach positiv,

(b) bestimmte Branchen werden aufgrund der überlegenen Importkonkurrenz schrumpfen, z. B. Textilproduktion und Teile der Landwirtschaft (= schrumpfende Produzentenrenten),

(c) afrikanische Verbraucher werden pro tanto gewinnen,

(d) Zollverluste für den Fiskus der Vertragsstaaten werden erheblich sein und sollten von der EU ausgeglichen werden, obwohl es in dieser Frage eine für das Ergebnis relevante Kontroverse über die richtige baseline gibt. 
Grundsätzliche Kritik an dieser Art Wirkungsanalyse wird aus zwei Richtungen vorgebracht. Zum einen ist eine anspruchsvolle CGE-Modellierung im Lichte der neueren Debatte über die Qualität afrikanischer Wirtschaftsstatistiken fragwürdig - das ist „Africa's statistical tragedy“ (Devarajan, Jerven). Diese Kritik kann kaum ausgeräumt werden. Zum anderen wird auf die Vielzahl von arbiträren Annahmen - Elastizitäten aus anderen Weltregionen etc. - verwiesen, die in die Modellierung eingehen. Armington ist ein gern gesehener Gast in dieser Runde. Dem begegnen die Studien korrekterweise mit Sensitivitätsanalysen, die es ermöglichen, wenigstens einen Teil der Annahmen besser abzuschätzen.

Eine grundsätzliche methodische Beschränkung der Wirkungsuntersuchungen bleibt dabei jedoch unterbelichtet. Die angesprochenen Modelle können die Wirkung von Zoll- und Quotenabbau auf existierende Branchen testen, aber sie können keine entgangene Industrialisierung messen - „industries that never were“ - also Branchen, die sich in einem vollständig liberalisierten Umfeld gar nicht in Afrika ansiedeln werden. Darauf aber bezieht sich neben den Folgen in der Landwirtschaft die Hauptkritik am EU-Verhandlungsansatz.

Man kann darüber räsonieren, warum der Sachverhalt entgangener Produktionsmöglichkeiten in den Wirkungsuntersuchungen nicht erscheint. Fehlende Rechenbarkeit ist sicher ein Faktor. Ausschlaggebend wird auch das erkenntnisleitende Grundmodell von Handelsvorteilen und Nachteilen unter Zollbedingungen sein, also das paradigmatische Bild von monoton sinkenden Skalenerträgen mit vollständiger Konkurrenz und „deadweight losses“ unter Zollschutz, bekannt als „monumentally unrealistic, [but] pedagogically convenient“ um RTAs zu studieren (Baldwin \& Wyplosz). Darin dominiert die Annahme von Handelsumlenkung zu globalen, hier: EU-Anbietern, die unter liberalen Handelsbedingungen effizienter als lokale Anbieter sind. Abbau von negativen Konsumentenrenten erscheint dann gern als Armutsbekämpfung. Eine Suchrichtung, wie skaleneffiziente Massenproduktion in Afrika unter ,smarten` Förderbedingungen allererst hergestellt werden kann, wird wissenschaftlich kaum angeboten.

Gleichwohl haben sich afrikanische Verhandler mit ihrer, sagen wir heuristischen Behauptung von Schutz- und Förderinstrumenten, die dem Zoll- und Quotenabbau auf ihrer Importseite entgegenwirken, zum Teil durchgesetzt. Sie haben den konventionellen Studien einfach nicht getraut. Aufgrund ihrer Initiative, nicht aufgrund höherer Einsicht europäischer Handels- oder Entwicklungspolitiker, wurden eine Reihe von zusätzlichen oder WTO-üblichen Special and Differential Treatment (SDT)-Klauseln in die EPA-Entwürfe einkopiert, oft allerdings in abgeschwächter Fassung. EU-Kommission und BMZ als Stellvertreter in der deutschen Debatte verweisen zu ihrer Verteidigung gern auf die Batterie dieser Safeguards in den EPAs. Dazu zählen sog. Infant Industry - Schutzklauseln, die mehr oder weniger explizit in allen regionalen EPAs (West-, Ost- und Südafrika) 
enthalten sind. Auch die Verteidigung von Exportsteuern und local content rules zur Förderung lokaler Weiterverarbeitung zählt dazu.

In der ursprünglichen Analyse dieses Autors hätten die Klauseln gerade noch hinreichenden Politikspielraum für intelligente Agrar- und Industrieförderung in Afrika gelassen, zumal wenn man bedenkt, dass kleinere Akteure im WTOMaßstab mit gewissen Regelüberschreitungen oft durchkommen. ${ }^{1}$ Im Ganzen sind die diversen Safeguards und Ausnahmeregeln jedoch umständlich gefasst und in ihrer vertraglichen Fassung vom Widerwillen der EU-Kommission geprägt, überhaupt Zugeständnisse machen zu müssen. Ihre Anwendung würde in den interessanten Fällen auch von der Zustimmung der EU-Seite abhängig sein. Der policy space für Afrika blieb in den Entwürfen also begrenzt. Das hat die Regierungen vor allem von Nigeria und Tansania dazu gebracht, die Unterschrift unter die Verträge $z u$ verweigern. Sie verweisen insbesondere darauf, dass durch die EPAs ihre Industriepolitik untergraben werde. Die Kritik wäre politisch überzeugender, wenn sie nicht in der letzten Minute vorgetragen würde und wenn diese Länder tatsächlich eine moderne Industriepolitik nach mittlerweile etablierten Qualitätskriterien hätten. Gleichwohl sind die Einwände im Kern richtig.

Das geographische Ergebnis ist ein Desaster. Die Landkarte der EU-Handelsregime in Afrika gleicht einem Flickenteppich und die wirtschaftlichen Regionalgemeinschaften Afrikas werden fragmentiert. In Kraft sind nur Abkommen mit einzelnen Mitgliedsstaaten afrikanischer Regionalgemeinschaften (Elfenbeinküste, Ghana, Kamerun, ähnlich Kenia) sowie mit zwei Teilgruppen der SADC ein eigentlicher Sündenfall für eine EU, die behauptet Regionalintegration in Afrika zu fördern. Die EU-Kommission, hier: die DG Trade, verweigert sich einer materiellen Nachbesserung der EPAs ungefähr so hartnäckig wie die deutsche Autoindustrie einer Hardware-Nachrüstung von Dieselfahrzeugen. Der pointierte Vergleich ist keineswegs hergeholt, weil er politischen Handlungsbedarf an höchster Stelle signalisiert.

Zwei Auswege bieten sich an. Mit relativ geringem Aufwand könnten entscheidende Schutzregeln und Steuerungsmechanismen in den EPA-Texten nachgebessert und afrikaweit vereinheitlicht werden, um (a) Nigeria, Tansania, Uganda usw. die Zustimmung zu erleichtern, (b) die Regionalgemeinschaften als Verhandlungspartner wieder herzustellen und (c) den ganzen Prozess auch für

1 Ein gutes Beispiel sind Länder in West- und Zentralafrika, die die Einfuhr von gefrorenen Hühnerteilen ganz untersagt oder über Lizenzen stark eingeschränkt haben. Kamerun zum Beispiel hat sich zum Zeitpunkt des Einfuhrverbots auf die europäische Hühnerpest berufen und die Sperre auch dann aufrechterhalten, als der Grund längst entfallen war. Dem wichtigeren Akteur Südafrika lässt der globale Norden, hier die USA, vergleichbare Einfuhrhürden für die gleiche Produktgruppe nicht durchgehen. 
afrikanische Länder interessant zu machen, die sich bislang komplett verweigern. Dazu zählen neben den genannten Regelungen viel zu starr gefasste Sensitive Products Lists, die Meistbegünstigungsklauseln und Ursprungsregeln. Auch könnte der Anteil der afrikanischen Importe, die überhaupt liberalisiert werden müssen, abgesenkt werden, ohne WTO-Konformität zu verletzen, was gegen Schluss der Verhandlungen sogar eine Reihe europäischer Regierungen (nicht die Bundesregierung) von der EU-Kommission verlangt hatten.

Als Alternative wird ein umfassendes Handelsabkommen mit dem neuen afrikanischen Continental Free Trade Area (CFTA) als Ersatz für die kritisierten EPAs diskutiert. Allerdings wird ein solcher Verhandlungsweg auch in der neuesten Publikation der EU zu ihrer Afrika-Strategie realistischerweise auf die lange Frist verschoben. Das CFTA wird etliche Jahre benötigen, um überhaupt eine Freihandelszone zu werden, und eine Zollunion wird CFTA nie werden, was ein entsprechendes Abkommen mit der EU technisch nahezu verunmöglicht. In dieser Frage herrschen politische Ratlosigkeit und Stillstand. Dieser Autor wird beidem, dem Mangel an handelstheoretischem Rat wie der politischen Blockade mit einer Publikation „Regional Integration, Trade and Industrialization in Africa“ (forthcoming 2019) entgegenzuwirken suchen.

Drittens: Die Dominanz landwirtschaftlicher Interessen in Brüssel hat seit jeher die Einsicht verhindert, dass afrikanische Bauern und Fischer faire Handelsbedingungen und eine wenigstens einigermaßen vergleichbare Förderung wie in der EU benötigen, um wirtschaftlich mithalten zu können. Die Forderung von Entwicklungsminister Müller nach fairem statt vollkommen freiem Handel geht exakt in diese Richtung. Im Rahmen der laufenden Generalüberholung der EU-Afrika-Beziehungen (sog. Post-Cotonou-Prozess) muss diese Frage in einer umfassenden Korrektur münden. Dazu gibt es in der EU trotz vorhandener wissenschaftlicher Expertise offiziell keine Bereitschaft; das verabschiedete Verhandlungsmandat schaut ganz anders aus. Auch die konzeptionelle Intransigenz der EU-Kommission im Rahmen der Diskussionen um die für 2020 anstehende Reform der Gemeinsamen Agrarpolitik (CAP) zeugt davon. Globalisierungskritiker fordern verstärkt die Abschaffung der EU-Agrarsubventionen. Als politische Maximalforderung ist das wissenschaftlich im Grunde nicht zu widerlegen; realpolitisch erscheint die Forderung vollkommen unrealistisch, nicht zuletzt, weil diese Direktzahlungen zusammen mit den Regional- und Strukturfonds in Europa Zentrifugalkräfte abbremsen, die in jeder Wirtschaftsgemeinschaft auftreten - im Übrigen auch in Afrika. Worin kann eine realistische Alternative bestehen?

Wer sich der Frage konzeptionell zu nähern versucht, macht die unerfreuliche Entdeckung, dass sich auf diesem Teilgebiet ein ähnlicher Bruch zwischen fundierter Kritik aus entwicklungspolitischer Sicht und Beiträgen der Wirtschaftswissenschaft, hier: der Agrarökonomie, etabliert hat wie oben zu den 
Handelsverträgen. Allen Diskutanten ist gegenwärtig, dass die EU seit einigen Jahren keine direkten Exportsubventionen mehr zahlt und insofern auch mit afrikanischer Landwirtschaft nicht unmittelbar unfair konkurriert. Von dort bis zur These der vollkommenen Wirkungslosigkeit von EU-Agrarsubventionen ist es aber ein weiter intellektueller Weg. Genau dies wird nun coram publico behauptet. Allenfalls wird (a) eine pfadabhängige Wirkung im strikten Sinn von Hysterese (= Weiterwirken bei Wegfall der Ursache) und (b) auf dem Umweg über die Beeinflussung von Weltmarktpreisen zugelassen. Diese Argumentationen können nicht überzeugen. Die in Frage stehende Subventionsinzidenz ist wie alle Fragen fiskalischer Inzidenz kompliziert, und im Maße wie die gegenwärtigen ,entkoppelten' Zahlungen eher Faktorpreise umschichten, z. B. eine Erhöhung der Bodenrente bewirken, anstatt Produktionsmengen zu erhöhen, verpufft sicher ein Teil der Subventionen ebenso wie die angestrebte ökologische Steuerungswirkung. Aber ein essentieller Teil der Subventionswirkung auf die europäische Handelsposition verbleibt, soweit absehbar.

Diskutieren wir eine mögliche politische Lösung am Beispiel der Baumwollproduktion in Afrika, die nicht nur aufgrund der EU-Subventionen, sondern vor allem aufgrund der massiven Ausgleichszahlungen durch den US Farm Act unter systemischem Druck steht. In den Ländern der sog. Cotton 4 - Gruppe in Westafrika (Benin, Burkina, Mali, Tschad) leben etwa eine Million Bauern vom Baumwollanbau. Diese Bauerngruppe über die in allen vier Ländern funktionierenden Aufkaufsysteme mit der gleichen Subvention pro Hektar auszustatten wie die Konkurrenz des Nordens, würde den EU-Entwicklungshaushalt nicht sprengen. Ähnliches gilt etwa für bestimmte Gruppen von Gemüsebauern. Fehlallokationen sind hier sogar weniger wahrscheinlich als bei der europäischen Konkurrenz. Der langfristig wünschenswerte Effekt wäre, alle Subventionssysteme abgestimmt herunterzufahren, wenn auch wichtige Drittanbieter mitspielen. Es liegt auf der Hand, solche Ausgleichszahlungen an Bauerngruppen in Afrika mit der Förderung ökologisch nachhaltigen Ackerbaus zu verbinden - im Zweifel wirksamer als in den beiden Säulen der CAP-Finanzierung.

Der Grabenbruch zwischen Politik und Theorie nimmt hier aber eine weitere Wendung. Wahlweise stützen beteiligte Ökonomen seit Jahren die These, wonach europäische Agrarexporte zur Ernährungssicherung in Afrika beitragen und auch insoweit nicht kritisiert werden sollten. Die These wird auch im Rahmen der ex ante EPA-Evaluierungen vorgebracht. Das ist im theoretischen Kern nichts als eine Anwendung des Theorems von der positiven Konsumentenrente unter Freihandelsbedingungen. Sie ist in zweifacher Weise angreifbar. Erstens unterstellt sie, dass europäische Produzenten effizienteste Anbieter unter Marktbedingungen sind. Das ist nicht nur wegen der CAP-Subventionen, sondern auch für Zucker oder in speziellen Konstellationen wie der des legendären Kuppelprodukts 
Hühnerschenkel fraglich, dessen eigene Produktionskosten artifiziell gegen Null gehen und das daher tiefgefroren günstig nach Afrika exportiert werden kann.

Zweitens enthält die These weitreichende Annahmen über - niedrige Angebotselastizitäten der afrikanischen Landwirtschaft unter verbesserten Handelsbedingungen, im Grunde über geringe Chancen von Agrarmodernisierung in Afrika. Es ist positiv zu vermerken, dass sich die Diskussion in jüngerer Zeit differenziert. Nicht zuletzt sorgfältige Produkt-Studien im Auftrag der GIZ lassen erkennen, dass bestimmte agrarische Wertschöpfungsketten in der Tat unter Zollschutz aufblühen. Das gilt exemplarisch für die angesprochene Geflügelzucht. Für bestimmte Produkte und in bestimmten Ländergruppen (Milch im Sahel) erscheinen die Entwicklungschancen der Produkt-/Kühlkette auf absehbare Zeit so ungünstig, dass Importe aus der EU (im Beispiel: Milchpulver) wenig Verdrängung von Primärproduktion, dafür aber neue Formen der Weiterverarbeitung im afrikanischen Land erlauben. Das betrifft auch die wachsende Weiterverarbeitung von Rohkakao zu Schokolade in der Elfenbeinküste und Ghana. Die Diskussion ist noch nicht abgeschlossen, zeigt aber eine wissenschaftlich wie entwicklungspolitisch akzeptable Suchrichtung.

Letztlich muss die gesamte Debatte im Zusammenhang mit den neuen strategischen Überlegungen zur Investitionsförderung in Afrika geführt werden, die sowohl bilateral (in Deutschland) als auch auf multilateraler Ebene (EU, G20) angestellt werden. Auch neue Investitionsförderung wird ohne einen reformierten handelspolitischen Kontext für Afrika nicht funktionieren. 